\title{
An Objective Comparison Between Gray Weighted Distance Transforms and Weighted Distance Transforms on Curved Spaces
}

\author{
Céline Fouard and Magnus Gedda \\ Centre for Image Analysis, Uppsala University, \\ Lägerhyddsvägen 3, SE-75237 Uppsala, Sweden \\ \{celine, magnusg\}@cb.uu.se
}

\begin{abstract}
In this paper, we compare two different definitions of distance transform for gray level images: the Gray Weighted Distance Transform (GWDT), and the Weighted Distance Transform On Curved Space (WDTOCS). We show through theoretical and experimental comparisons the differences, the strengths and the weaknesses of these two distances.
\end{abstract}

\section{Introduction}

Automatic image analysis processes are generally performed on binary images. However, when images are acquired, gray level values have specific meanings. In some images, they can represent a third (fourth) dimension for 2D (3D) images, or they can represent blurry boundaries of objects, or the object density distribution and many other features. In all the cases, the binarization process, although often mandatory to perform further automated image analysis, results in a loss of information.

To overcome this problem, more and more methods are proposed to perform image analysis directly on gray level images [1,2, 3. This is also the case of distance transforms which are widely used on binary images to extract shape and size information [4].

Rutovitz first proposed in [5] a Gray Weighted Distance Transform (GWDT) which uses a pixel gray value as a cost to traverse this pixel. The Gray Weighted Distance (GWD) between two pixels is then defined as the smallest weighted sum of gray level values along the discrete path between these two points. Levi and Montanari also proposed in [6] a distance transform on gray level images where the length of each path is weighted by the gray values of the pixels along the path. In their definition, the length of a path is defined as the discretization of the integral of the pixel values along the path. Saha et al. 7] proposed a theoretical framework and a dynamic programming method for the $n$-dimensional computation of the Gray Weighted Distance Transform. Verbeek and Verwer 8 and Kimmel et al. 9] used this Gray Weighted Distance Transform to solve the eikonal equation.

Another way of computing distance transforms on gray level images was proposed by Toivanen in [10. The path between two points is then defined as a 
$n+1$ dimensional path constraint to lie on the hyper-surface defined by the gray level values (here considered as heights on the $n$ dimensional image). This distance transform is thus called Weighted Distance Transform On Curve Space (WDTOCS).

Other distances have been defined on gray level images seen as supports for fuzzy sets. For example, Bloch detailed in [11] several distances between fuzzy sets. She also proposed a new geodesic distance for fuzzy sets [12. Soille [13] also defined a geodesic measure for fuzzy sets inspired by Levi and Montanari's definition [6].

In this paper, we focus only on distance transforms, so the later distance definitions are beyond the scope of this study. As we want the path defined on gray levels to be able to reach the background, we will not consider geodesic distances either. Our aim is to understand how the two different distance transform definitions ; Gray Weighted Distance (GWD) and Weighted Distance On Curve Space (WDCOS), behave on gray level images where the gray level values have different meanings. We first propose a theoretical comparison based only on their definition and mathematical properties in Section 2. We then compare the results obtained by using these definitions to compute the radius of a fuzzy disk in Section 3. The comparison is then performed on density maps (Section 4) and height maps (Section 5).

\section{Comparison from Definitions}

In this paper, we consider gray level images $\mathcal{I}: \mathbb{Z}^{n} \longrightarrow \mathbb{R}$ as functions from the discrete points of the $n$-dimensional space $\mathbb{Z}^{n}$ to the space of real numbers $\mathbb{R}$. The gray level values correspond either to heights or to fuzzy membership functions. The notion of height comes with $2 \mathrm{D}$ images where the third dimension (elevation) is coded with gray level values in the image. A fuzzy membership function is defined as a mapping to the interval of real numbers $[0,1]$. The notions of background and foreground can be extended to gray level images as follows: $B=\left\{p \in \mathbb{Z}^{n} \mid \mathcal{I}(p)=0\right\}$, and $F=\left\{p \in \mathbb{Z}^{n} \mid \mathcal{I}(p)>0\right\}$. In the case of a fuzzy image, the several gray levels of the foreground pixels can be seen as their belonging degree of the object. In the case of height maps, the gray level values correspond to the altitude of the ground.

\subsection{Gray Level Distance Maps}

A distance map is generally defined on a crisp image but can be extended to gray level images:

Definition 1 (Distance map). Given a gray level image $\mathcal{I}$, the distance map of $\mathcal{I}: \mathcal{D}_{\mathcal{I}}$ is a gray level image where the value of each point of the foreground corresponds to its shortest distance to the background.

Given a distance definition $d$, a point $p \in F$ of the foreground and a point $q \in B$ corresponding to its nearest background point, $\mathcal{D}_{\mathcal{I}}(p)=d(p, q)$. In the case of a 
crisp image, $d(p, q)$ only depends on the length of the path $\mathcal{P}_{p q}$ between $p$ and $q$. This path can be either continuous as in Euclidean Distance Transforms [14] or discrete, as in the chamfer algorithm [15]. In the case of gray level distance maps, $\mathcal{P}_{p q}$ is a path between $p$ and $q$ lying on the hyper-plane defined by the gray level values of $\mathcal{I}$. In the following, we consider the continuous function $\pi:[0,1] \longrightarrow \mathbb{R}^{n}$ following $\mathcal{P}_{p q}$ such that $\pi(0)=\mathcal{I}(p)$ and $\pi(1)=\mathcal{I}(q)$. The length of $\mathcal{P}_{p q}$ depends not only on the spacial distance between $p$ and $q$, but also on the gray level values along the path. As digital gray level images are defined on discrete grid, the gray level values along a continuous path may not bee known. This is why, even if they are theoretically defined in the continuous space $\mathbb{R}^{n}$, both GWDT and WDTOCS are practically computed on discrete paths. In this case, each step $\left[t_{i}, t_{i+1}\right]$ of the discrete path with $t_{0}=p$ and $t_{m}=q$ is attributed a cost value $w_{i}$ depending on the length $\left\|t_{i}-t_{i+1}\right\|$ of the step $i$, and of the gray level values $\mathcal{I}\left(t_{i}\right)$ and $\mathcal{I}\left(t_{i+1}\right)$. The global cost of the path is the sum of all the costs of the local steps: $\mathcal{W}\left(\mathcal{P}_{p q}\right)=\sum_{i} w_{i}$, and the final distance between $p$ and $q$ is the minimum of the costs of all the paths: $d_{p q}=\min \left\{\mathcal{W}\left(\mathcal{P}_{p q}\right)\right\}$.

\subsection{GWD and WDOCS Definitions}

Continuous Case. The Gray Weighted Distance is defined in the continuous case by [6] and [7] as follows: $\mathcal{D}_{G W D T}=\int_{0}^{1}|\pi(t)| d t$. It corresponds to the surface area estimation under the curve path $\mathcal{P}_{p q}$. The Weighted Distance On Curved Space is defined in [10] as the length of the shortest geodesic path $\mathcal{P}_{p q}$ between $p$ and $q$. It is expressed as follows in the continuous case: $\mathcal{D}_{W D T O C S}=$ $\int_{0}^{1}\left|\frac{d \pi}{d t}(t)\right| d t$. Figures of the first line of table 1 illustrate these two definitions.

Discrete Case. In the discrete case, the cost of each step for GWD and WDOCS are respectively:

$$
\begin{aligned}
\text { GWD: } \quad w_{G W D_{i}} & =\frac{1}{2}\left(\mathcal{I}\left(t_{i}\right)+\mathcal{I}\left(t_{i+1}\right)\right) \times\left\|t_{i}-t_{i+1}\right\| \\
\text { WDOCS: } w_{W D O C S_{i}} & =\sqrt{\left(\mathcal{I}\left(t_{i+1}\right)-\mathcal{I}\left(t_{i}\right)\right)^{2}+\left\|t_{i}-t_{i+1}\right\|^{2}}
\end{aligned}
$$

In both cases, the spatial distance between two steps $\left\|t_{i}-t_{i+1}\right\|$ can be either the Euclidean distance: $\left\|t_{i}-t_{i+1}\right\|=\left\{\begin{array}{l}1 \text { if } t_{i} \text { and } t_{i+1} \text { are } 4 \text {-neighbors } \\ \sqrt{2} \text { if } t_{i} \text { and } t_{i+1} \text { are strict } 8 \text {-neighbors }\end{array}\right.$ or Borgefors [15] optimal propagating weights for a binary $3 \times 3$ mask, i.e.

$\left\|t_{i}-t_{i+1}\right\|=\left\{\begin{array}{l}0.95509 \text { if } t_{i} \text { and } t_{i+1} \text { are } 4 \text {-neighbors } \\ 1.36930 \text { if } t_{i} \text { and } t_{i+1} \text { are strict } 8 \text {-neighbors }\end{array}\right.$

Table 1 summarizes the different mathematical properties of these two definitions. The last line of this table considers the metric properties (definitivity, positivity, triangular inequality and positive homogeneity) of the two distances.

\subsection{About Unit Consistency}

By considering gray level values as a $n+1$ image dimension, WDTOCS mixes spatial and intensity units. This may raise several problems. A practical one for 
Table 1. Theoretical comparison of GWD and WDOCS

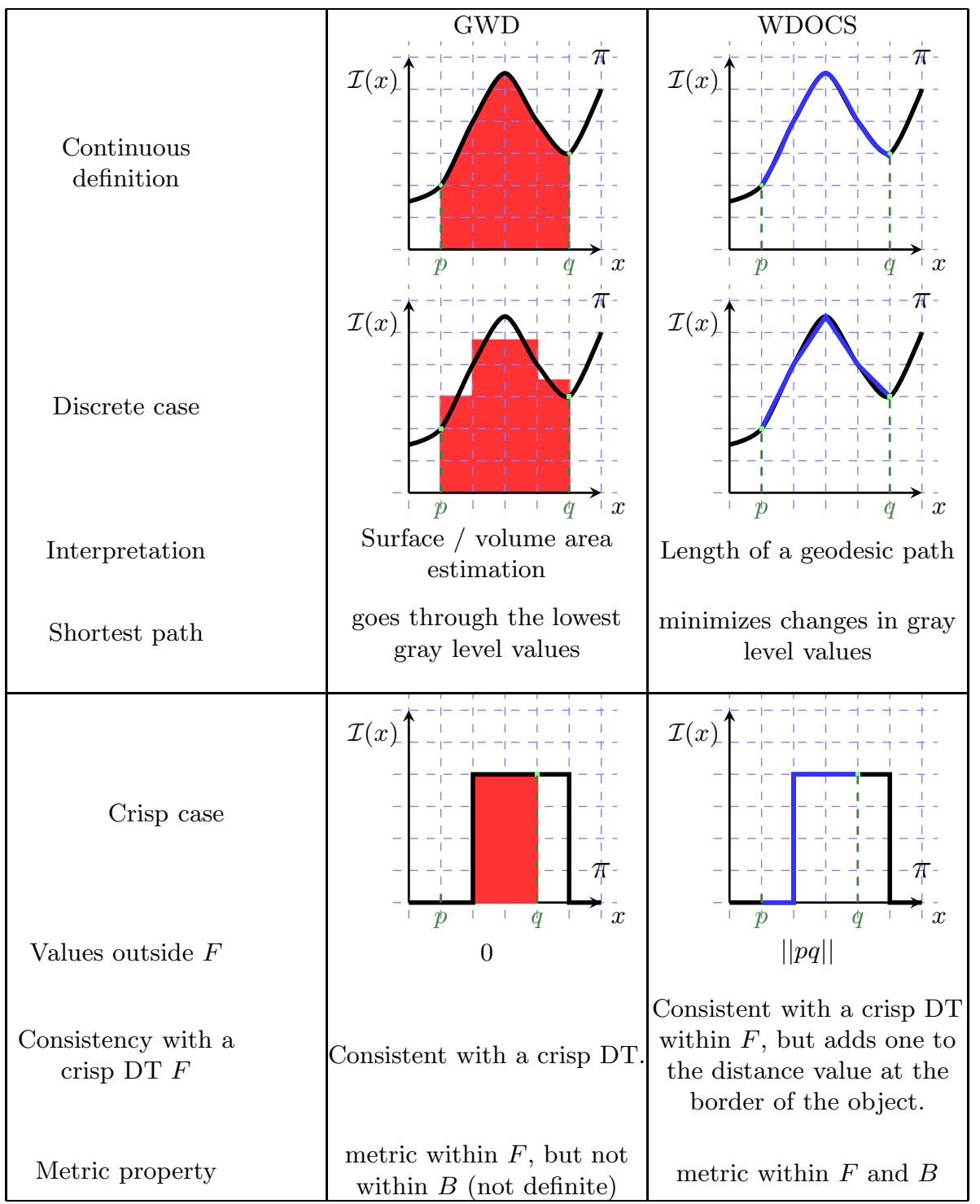

example occurs when the gray level values are small with respect to the image spatial dimensions: the WDTOCS makes no difference with the binary DT as illustrated Figure 1. The experiments used in this paper to illustrate the different behaviors of WDTOCS and GWDT are performed on 2D images to allow full display of the results. Let denote the spatial dimensions of the images along $x$ 
and $y$ directions $\mathcal{I}_{x}$ and $\mathcal{I}_{y}$ respectively. To overcome this problem in Sections 4 and [5, we scale the gray level values between 0 and $\mathcal{I}_{\text {max }}=\frac{\mathcal{I}_{x}+\mathcal{I}_{y}}{2}$ to make them comparable to the spatial dimensions (Figure 1). This unit issue does not affect GWDT as it does not mix spatial and gray level units. On the other hand, it considers the integration of distance values, which is more often associated with surface area estimation than with distances.

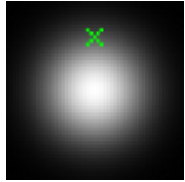

(a)

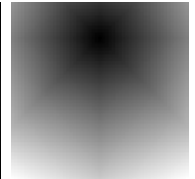

(b)

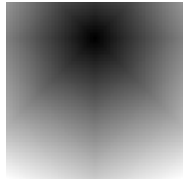

(c)

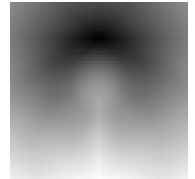

(d)

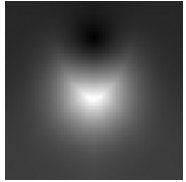

(e)

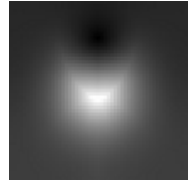

(f)

Fig. 1. (a) Original Gauss image with transform start point marked. (b) Chamfer distance transform from the marked point. (c) WDTOCS using $\mathcal{I}_{\max }=1$. (d) WDTOCS using $\mathcal{I}_{\text {max }}=\frac{\mathcal{I}_{x}+\mathcal{I}_{y}}{2}$. (e) GWDT using $\mathcal{I}_{\text {max }}=1$. (f) GWDT using $\mathcal{I}_{\text {max }}=\frac{\mathcal{I}_{x}+\mathcal{I}_{y}}{2}$.

\subsection{Implementation}

Both WDTOCS and GWDT are discrete path based distance transforms. They are computed with the principle of the chamfer algorithm. In [10] Toivanen proposed to compute WDTOCS by iterating Rosenfeld's raster scans [16] until stability. Saha et al. 7] and Ikonen [17] proposed a wave-front propagation implemented through a pixel-queue algorithm which starts from the border points and propagates local distances to the center of the object.

We implemented both methods (Rosenfeld's raster scans until stability and wave-front propagation algorithms) for both GWDT and WDTOCS and the numerical and visual results for the two different implementation methods are exactly the same. On small images, the calculation time is almost the same, but for larger images, the wave-propagation algorithm is more efficient.

\section{Measurement of Continuous Disks Radii}

The radius of a discrete disk is obtained by taking the highest value of the distance map computed inside the disk. We produce

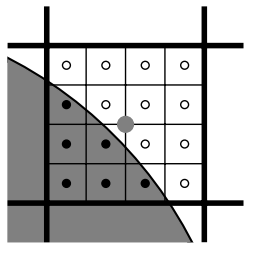

Fig. 2. digital fuzzy disks which are discretization of continuous disks and compare the results obtained by WDTOCS and GWDT with Euclidean distance transform and chamfer distance transform on a binarization of these fuzzy disks. The value of pixels within a fuzzy disk is 1 , and 0 for a pixel outside. For border pixels, the value is calculated by subsampling the considered pixel as suggestion in [18. Figure 2 shows an example of a pixel subsampled in 16 pixels whose value is $\frac{6}{4^{2}}$. 
We produce such fuzzy disks with several real radii and compute:

— WDTOCS on the gray level fuzzy disks. As we saw in previous section, WDTOCS crates a step of value one between inside and outside pixels. To compute fuzzy disks radii, we thus remove one to the final radius value.

— GWDT on the gray level fuzzy disks

- chamfer distance transform on a binarization of the gray level fuzzy disks with a threshold at 0.5

_ Euclidean distance transform [14] on a binarization of the gray level fuzzy disks with a threshold at 0.5

Figure 3 (a) shows the results obtained for radii from 2 to 10 pixels, and figure 3 (b) shows a close up of these curves for radii between 2 and 3 pixels.

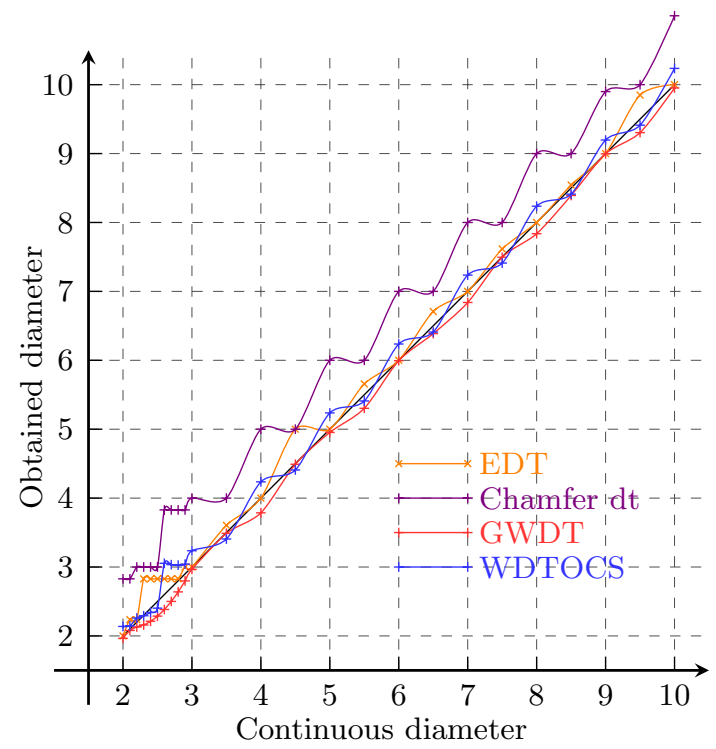

(a) Results for radii from 2 to 10 pixels

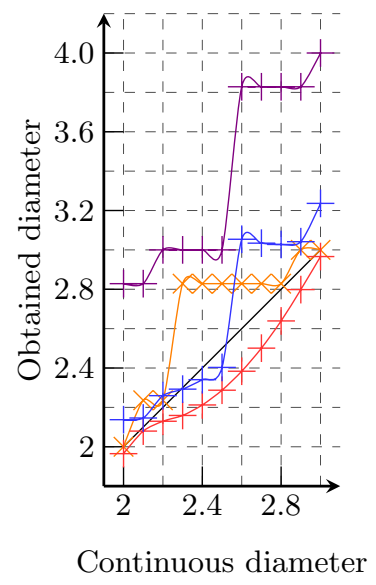

(b) Close up of (a) curves for radii from 2 to 3 pixels

Fig. 3. Radii of digitized fuzzy disks obtained with Euclidean DT (orange), Chamfer DT (purple), WDTOCS (blue) and GWDT (red)

We can see in Figure 3 (a) that WDTOCS and GWDT produce radii which are close to the real ones; at least closer to those given by the weighted distance on the threshold disk. Figure 3 (b) shows that for small radii, the two gray level distances give better results than the Euclidean DT on threshold disks. Generally, GWDT tends to underestimate radii as WDTOCS sometimes underestimate or overestimates radii.

${ }^{1}$ EDT code courtesy of David Coeurjolly http://www.cb.uu.se/ \{\}tc18/ 


\section{Comparison on Density Maps}

In many areas imaging devices produce density maps, e.g. medical imaging. In these cases gray level distance transforms can be used when calculating density based distances. An example application is content based clustering of local maxima in electron tomography images of proteins [19]. In this example application, it is desirable to get high distance values when measuring distances between points in different parts of the proteins (i.e. in different high density blobs), and low distance values when measuring distances between points in the same part.

In the following, we compute point-to-point distance by computing distance transforms (GWDT or WDTOCS) from a starting point and back-tracking the path to the second point. To compare GWDT and WDTOCS for point-to-point distance measures in density maps, we use a synthetic image of two gray level blobs and a real image slice of a protein obtained by electron tomography. In both case we compute

- a distance $d_{\text {within }}$ between two points of the same blob

- a distance $d_{\text {trans }}$ between two points taken in two different blobs

- the ratio $r=\frac{d_{\text {trans }}}{d_{\text {within }}}$ which gives an indication of the effectiveness measure of the delineation of two density blobs (i.e. the larger $r$ is, the better we can differentiate the two different blobs.)

Synthetic Image. The synthetic image is a $40 \times 408$-bit image which consists of two high-density objects. It is then inverted to map high densities to low gray level values. In Fig. 4 the gray level distance paths, and the respective distance transforms, are shown. The values of $d_{\text {within }}, d_{\text {trans }}$, and $r$ are listed in Table 2

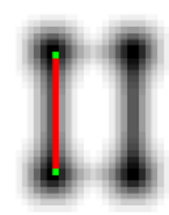

(a)

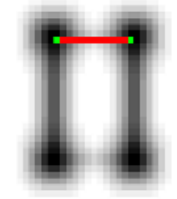

(b)

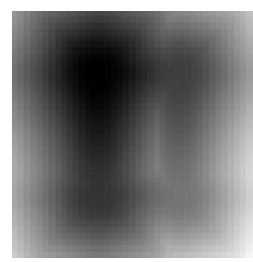

(c)

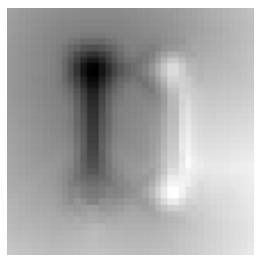

(d)

Fig. 4. (a) Inverted synthetic image with the GWDT path (red) and WDTOCS path path (blue) for the measure of $d_{\text {within }}$ (paths are overlapping). (b) Paths corresponding to the measure of $d_{\text {trans }}$ (c) Corresponding GWDT. (d) Corresponding WDTOCS.

Real Image. The real image is a $36 \times 36$ 8-bit image, with a pixel size of 5.24 Angström, and is taken from a slice of a $51 \times 51 \times 51$ protein density volume imaged using electron tomography. The slice shows the inertials of two blobs interconnected with lower gray levels. In Fig. 5 the gray level distance paths, and the respective distance transforms, are shown. In Table 3 the different distance values are listed. 
Table 2. Distance measures in synthetic image

\begin{tabular}{lrrr}
\hline Measure & $d_{\text {within }}$ & $d_{\text {trans }}$ & $r=d_{\text {trans }} / d_{\text {within }}$ \\
\hline GWDT & 187.72 & 225.38 & 1.20 \\
WDTOCS & 37.54 & 68.27 & 1.82 \\
Euclidean DT & 19 & 12 & 0.63 \\
\hline
\end{tabular}

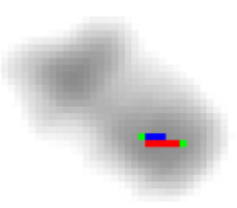

(a)

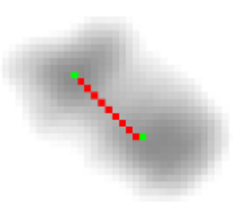

(b)

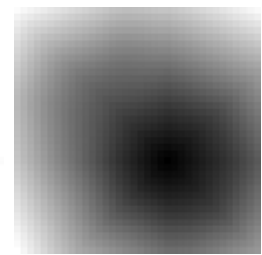

(c)

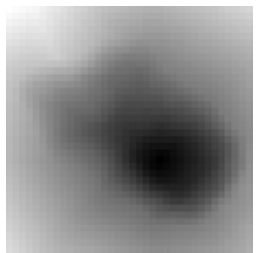

(d)

Fig. 5. (a) Inverted protein slice with the GWDT path (red) and WDTOCS path path (blue) for the measure of $d_{\text {within }}$. (b) Paths for the measure of $d_{\text {trans }}$ (paths are overlapping). (c) GWDT. (d) WDTOCS.

Table 3. Distance measures in protein slice image

\begin{tabular}{lrrr}
\hline Measure & $d_{\text {within }}$ & $d_{\text {trans }}$ & $r=d_{\text {trans }} / d_{\text {within }}$ \\
\hline GWDT & 125.13 & 303.40 & 2.42 \\
WDTOCS & 6.45 & 17.38 & 2.69 \\
Euclidean DT & 6.08 & 13.45 & 2.21 \\
\hline
\end{tabular}

In both the synthetic and the real cases, the fraction $r$ shows that both GWDT and WDTOCS allow to separate the two blobs better than the Euclidean distance (in the synthetic case the two points inside the same blob are further than the two points taken in two different blobs). In both the synthetic and the real cases, the blobs are better delineated in the case of WDTOCS as $r_{W D T O C S}>r_{G W D T}$ and we can also see that the corresponding distance transforms Fig. 4 and 5 also separate the two blobs better.

\section{Point-to-Point Distances in Height Maps}

In areas where height maps are common, e.g. remote sensing, fuzzy distance can be a valuable tool for calculating content-based distances in images. One application is shortest path-finding in terrain images 2 .

\footnotetext{
${ }^{2}$ Remark: In the case of heights maps, all altitude of the maps correspond to altitudes of the ground. Here we consider the 0 level as absolute. Thus, if the distances of two points of the same altitude are taken at different altitudes, this can lead to a shift in the GWD value.
} 
To compare GWDT and WDTOCS for point-to-point distance measures in height maps, we use a synthetic height map image and a height map image taken of the Grand Canyon.

Synthetic Image. The synthetic image is a $136 \times 1368$-bit image which consists of a large central ridge. The paths between two points on the ridge calculated with GWDT and WDTOCS, along with the corresponding distance transforms are shown in Fig. 6.

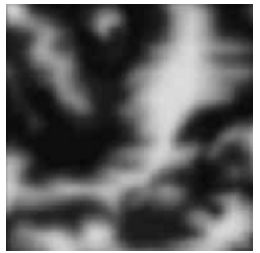

(a)

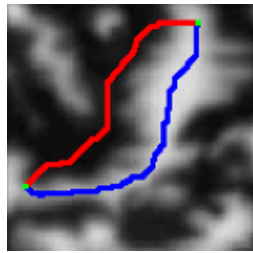

(b)

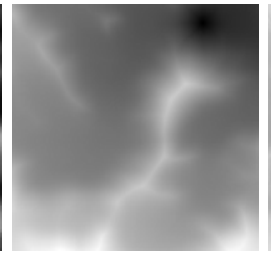

(c)

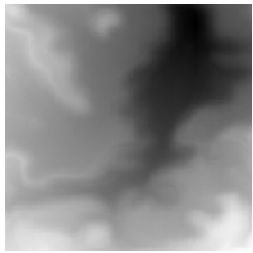

(d)

Fig. 6. (a) Original height map. (b) GWDT path (red) and WDTOCS path (blue) between two points on the ridge. (c) GWDT. (d) WDTOCS.

Fig. 6] shows the two different behaviors GWDT and WDTOCS: GWDT path goes through the lowest possible gray level values, and thus goes down from the ridge, and then up to reach the end point, while WDTOCS path minimizes the number of changes in gray level values, and thus remains on the ridge.

Real Image. The real image is a $2400 \times 1600$ 8-bit image, with a pixel size of 60 meters, and is taken from a a $4097 \times 2047$ height map of the Grand Canyon area. The image shows the canyon stretching from a lake in the left part of the image, and continuing as a fissure to the far right of the image. Each pixel unit ( 0 to 255 ) corresponds to 10.004 meters, and the pixel value 0 corresponds to a base elevation of 284 meters. In Fig. 7 a surface rendered representation, along with the fuzzy distance paths and the fuzzy distance transforms, are shown.

In this case, GWDT path follows the minimum gray level values as expected and remains in the bottom of the Grand Canyon. However, the WDTOCS path surprisingly goes out the canyon, and follows a high altitude plateau before going down to the canyon again. On the corresponding WDTOCS Fig. 7 (d), we can see, that the WDOCS values are low within the canyon until the middle of the image, and then begin to be higher. To better understand what happens in this case, we compute paths between points within this region as shown in Fig. 8 .

In this case, the first part of the WDTCOS path also goes away from the canyon center. This is due to the fact that the ground of this part is highly irregular as shown in Fig. 8 (c) and that WDTCOS path looks for places where the gray values vary more slowly. When the bottom ground values becomes regular again, the WDTCOS path follows the canyon. 


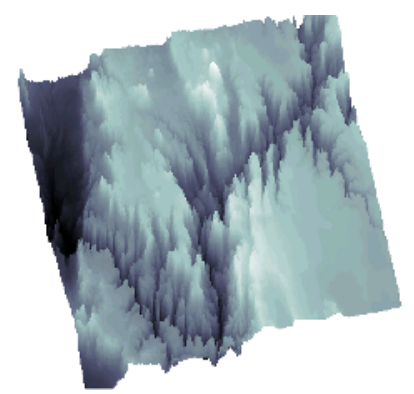

(a)

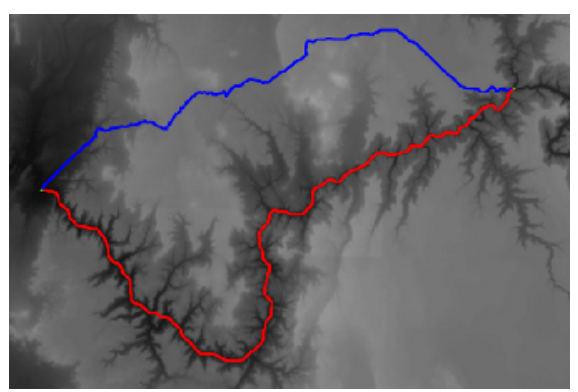

(b)

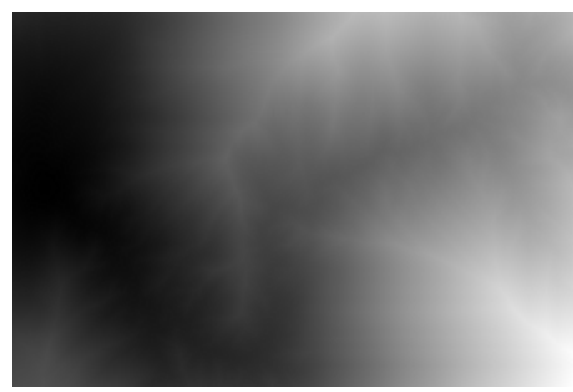

(c)

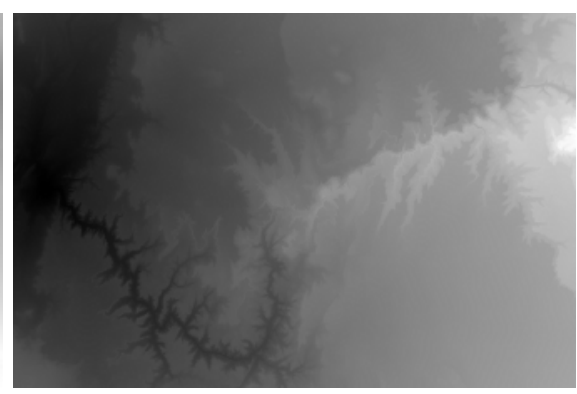

(d)

Fig. 7. (a) Grand Canyon height map surface rendered. (b) Height map with GWDT path (red) and WDTOCS path (blue) overlaid. (c) GWDT. (d) WDTOCS.

\section{Discussion and Conclusion}

In this paper, we compare two distance transform defined on gray level images. The Gray Weighted Distance (GWD) defines the length of a path as the spatial length of this path weighted by gray level values along this path. It can also be seen as the computation of the surface area delimited by the path as shown in Table 1. The Weighted Distance On Curved Space (WDOCS) on the other hand, defines the distance between two points as the length of the geodesic path lying on the hyper-surface defined by the gray level values. A GWD path will thus follow low gray level values whereas WDOCS paths will minimize the changes in gray level values.

The different experiments of this paper shows that the WDOCS depends highly on the scale of the gray level values, contrary to the GWD. In a general way, GWDT tends to smooth the values in the original gray level image, contrary to WDTOCS which tends to sharpen the differences between gray level values. This makes WDOCS more sensitive to the noise, whereas the GWD is more robust to local changes of gray level values. However, this also makes WDTOCS more accurate to delineate different gray valued objects in the same image.

The choice between these two distance transform definitions is highly application dependent. It depends on the aim of the application: either highlighting gray level differences or smoothing the values to obtain average distances. 


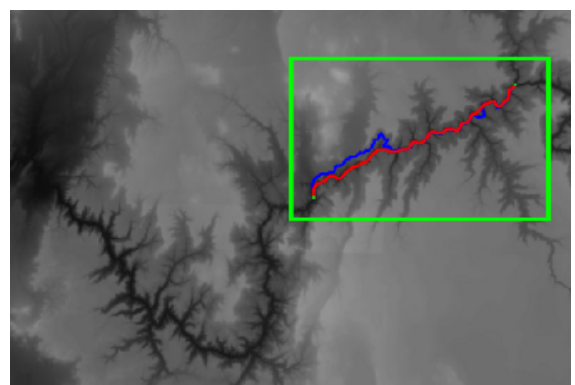

(a)

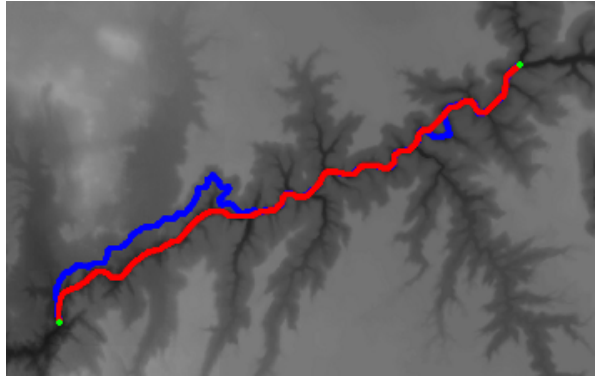

(b)

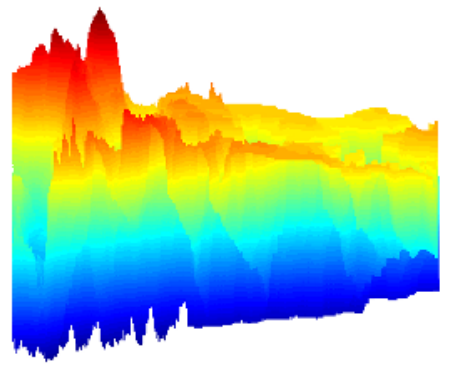

(c)

Fig. 8. (a) Height map with GWDT path (red) and WDTOCS path (blue) overlaid. (b) Close-up on paths showing WDTCOS path outside the canyon in the left part of the image. (c) The close up surface rendered and viewed sideways (from south) showing the jagged bottom of the left part of the canyon.

\section{Acknowledgments}

The authors would like to thank Dr. Natasa Sladoje and Dr Joakim Lindblad for their scientific support. The Grand Canyon data is obtained from The United States Geological Survey (USGS), with processing by Chad McCabe of the Microsoft Geography Product Unit.

\section{References}

1. Bloch, I.: Fuzzy spatial relationships for image processing and interpretation: a review. Image and Vision Computing 23(2) (2005) 89-110

2. Sladoje, N., Nyström, I., Saha, P.K.: Mesurements of digitized objects with fuzzy borders in 2D and 3D. Image and Vision Computing 23(2) (2005) 123-132

3. Arcelli, C., Luca, S.: Skeletoinization of labeled gray-tone images. Image and Vision Computing 23(2) (2005) 159-167

4. Borgefors, G.: Applications using distance transforms. In Arcelli, C., Cordella, L.P., Sanniti di Baja, G., eds.: Aspects of Visual Form Processing. World Scientific, Singapore (1994) 83-108 
5. Rutovitz, D.: Data structures for operations on digital images. In Cheng, G.C., ed.: Pictorial Pattern Recognition, Washington, Thompson (1968) 105-133

6. Levi, G., Montanari, U.: A gray-weighted skeleton. Information and Control 17(1) (1970) 62-91

7. Saha, P., Wehrli, F.W., Gomberg, B.R.: Fuzzy distance transform: Theory, algorithms, and applications. Computer Vision and Image Understanding 86(3) (2002) 171-190

8. Verbeek, P., Verwer, B.: Shading from shape, the eikonal equation solved by greyweighted sitance transform. Pattern Recognition Letters 11 (1990) 681-690

9. Kimmel, R., Kiryati, N., Bruckstein, A.: Sub-pixel distance maps and weighted distance transforms. Journal of Mathematical Imaging and Vision 6 (1996) 223-233

10. Toivanen, P.J.: New geodesic distance transforms for gray-scale images. Pattern Recognition Letters 17(5) (1996) 437-450

11. Bloch, I.: On fuzzy distances and their use in image processing under imprecision. Pattern Recognition 32(11) (1999) 1873

12. Bloch, I.: Geodesic balls in a fuzzy set and fuzzy geodesic mathematical morphology. Pattern Recognition 33(6) (2000) 897-906

13. Soille, P.: Generalized geodesy via geodesic time. Pattern Recognition Letters $\mathbf{1 5}$ (1994) 1235-1240

14. Saito, T., Toriwaki, J.: New algorithms for euclidean distance transformations of an $n$-dimensional digitizd picture with applications. Pattern Recognition 27 (1994) $1551-1565$

15. Borgefors, G.: Distance transformations in digital images. Computer Vision, Graphics, and Image Processing 34 (1986) 344-371

16. Rosenfeld, A., Pfaltz, J.L.: Sequential operations in digital picture processing. Jornal of the ACM 13(4) (1966) 471-494

17. Ikonen, L.: Pixel queue algorithm for geodesic distance transforms. In et al., E.A., ed.: Proc. of 12th Int. Conf. on dgci. Volume 3429., Springer-Verlag (2005) 228-239

18. Sladoje, N., Lindblad, J.: Estimation of moments of digitized objects with fuzzy borders. In Springer-Verlag, ed.: Proc. 13th iciap. Volume 3617. (2005) 188-195

19. Gedda, M., Svensson, S.: Separation of blob-like structures using fuzzy distance based hierarchical clustering. In Georgsson, F., Börlin, N., eds.: Symposium on Image Analysis, SSBA 2006, Umeå, Sweden, March 16-17, 2006, Proceedings. (2006) $73-76$ 